\title{
Quantitative imaging: systematic review of perfusion/flow phantoms
}

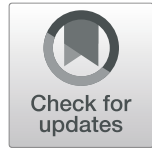

Marije E. Kamphuis ${ }^{1,2^{*}}$ (D, Marcel J. W. Greuter ${ }^{2,3}$, Riemer H. J. A. Slart ${ }^{3,4}$ and Cornelis H. Slump ${ }^{2}$

\begin{abstract}
Background: We aimed at reviewing design and realisation of perfusion/flow phantoms for validating quantitative perfusion imaging (PI) applications to encourage best practices.

Methods: A systematic search was performed on the Scopus database for "perfusion", "flow", and "phantom", limited to articles written in English published between January 1999 and December 2018. Information on phantom design, used PI and phantom applications was extracted.

Results: Of 463 retrieved articles, 397 were rejected after abstract screening and 32 after full-text reading. The 37 accepted articles resulted to address PI simulation in brain $(n=11)$, myocardial $(n=8)$, liver $(n=2)$, tumour $(n=1)$, finger $(n=1)$, and non-specific tissue $(n=14)$, with diverse modalities: ultrasound ( $n=11)$, computed tomography $(n=11)$, magnetic resonance imaging $(n=17)$, and positron emission tomography $(n=2)$. Three phantom designs were described: basic $(n=6)$, aligned capillary $(n=22)$, and tissue-filled $(n=12)$. Microvasculature and tissue perfusion were combined in one compartment $(n=23)$ or in two separated compartments $(n=17)$. With the only exception of one study, inter-compartmental fluid exchange could not be controlled. Nine studies compared phantom results with human or animal perfusion data. Only one commercially available perfusion phantom was identified.

Conclusion: We provided insights into contemporary phantom approaches to PI, which can be used for ground truth evaluation of quantitative PI applications. Investigators are recommended to verify and validate whether assumptions underlying PI phantom modelling are justified for their intended phantom application.
\end{abstract}

Keywords: Microcirculation, Perfusion imaging, Phantoms (imaging), Reference standards

\section{Key points}

- Without a validated standard, interpretation of quantitative perfusion imaging can be inconclusive.

- Perfusion phantom studies contribute to ground truth evaluation.

- We systematically reviewed design and realisation of contemporary perfusion phantoms.

- Assessed phantom designs are diverse and limited to single tissue compartment models.

\footnotetext{
*Correspondence: m.e.kamphuis@utwente.nl

'Multimodality Medical Imaging M3i Group, Faculty of Science and Technology, Technical Medical Centre, University of Twente, PO Box 217, Enschede, The Netherlands

${ }^{2}$ Robotics and Mechatronics Group, Faculty of Electrical Engineering,

Mathematics, and Computer Science, Technical Medical Centre, University of

Twente, Enschede, The Netherlands

Full list of author information is available at the end of the article
}

- Investigators are encouraged to adopt efforts on phantom validation, including verification with clinical data.

\section{Background}

Perfusion imaging (PI) is a powerful method for assessing and monitoring tissue vascular status, and alterations therein. Hence, PI is generally aimed at distinguishing healthy from ischemic and infarcted tissue. PI applications cover various imaging modalities such as ultrasound, computed tomography (CT), positron emission tomography (PET), and magnetic resonance imaging (MRI) that can record perfusion parameters in a wide spread of tissues including brain, liver, and myocardial tissue. A distinction can be made between contrast-enhanced and non-contrast PI approaches. The pertinent signal intensity in tissue can be 
recorded as a function of time or after a time interval, called dynamic or static PI respectively. This systematic review focuses on dynamic PI, as this approach enables quantitative analysis and absolute quantification of perfusion. In dynamic PI, it is possible to construct mathematical models that fit image data with model parameters in order to explain observed response functions in tissue. For example, time-intensity curves highlight the distribution of contrast material into the tissue over time. Model outcomes include computation of absolute blood flow (BF), blood volume (BV), and/or mean transit times (MTTs) [1]. Multiple BF models of tissue perfusion exist, including model-based deconvolution, modelindependent singular value decomposition and maximum upslope models [2]. These BF models are increasingly used in addition to standard semiquantitative analysis, as these show potential towards better accuracy and standardised assessment of perfusion measures [3-5].

Without a validated standard, interpretation of quantitative results can be challenging. Validation and/or calibration of absolute perfusion measures is required to ensure unrestricted and safe adoption in clinical routine [6-8]. Validation approaches include in vivo, ex vivo, in vitro, and in silico studies and combinations hereof. Each approach has advantages and disadvantages, and may differ in level of representativeness, controllability of variables, and practical applicability. Our focus was on in vitro studies, i.e., physical phantom studies. Phantom studies contribute to ground truth evaluation of single aspects on quantitative PI applications in a simplified, though controlled, environment. Phantom studies also allow for the comparison and optimisation of imaging protocols and analysis methods. We hereby observe a shift from the use of static to dynamic perfusion phantoms (i.e., with a flow circuit), as the latter enables indepth evaluation of time-dependent variables.

In general, it can be challenging to translate findings from phantom studies into clinical practice. For example, it can be questionable whether certain choices and simplifications in perfusion phantom modelling are justified. Intra- and interdisciplinary knowledge sharing on phantom designs, experimental findings, and clinical implications can be used to substantiate this. Hence, this systematic review presents an overview on contemporary perfusion phantoms for evaluation of quantitative PI applications to encourage best quantitative practices.

\section{Methods}

A systematic search on general and contemporary perfusion phantoms was conducted using Scopus database online, which includes MEDLINE and EMBASE. The query included "perfusion", "flow", and "phantom". Inclusion was limited to English written articles and reviews published between January 1999 and December 2018.

Two investigators independently screened titles and abstracts (M.E.K. and M.J.W.G.), whereby in vivo, ex vivo and in silico related perfusion studies were excluded, even as non-related in vitro studies (e.g., static and large-vessel phantoms). We hereby note that thermal and optical PI techniques fall outside the scope of this review. The same investigators performed full-text screening and analysis. Study inclusion required incorporation of microvascular flow simulation and we excluded single-vessel phantom studies. In addition, references were scrutinised on cross-references. Observer differences were resolved by discussion.

The perfusion phantom overview concerns three main aspects regarding ground truth evaluation of quantitative PI, as schematically depicted in Fig. 1. Details on perfusion phantom design, studied PI application and overall phantom application were extracted from each paper. We categorised phantom design features in terms of simulated anatomy, physiology, and pathology. Anatomy simulation lists information on the studied tissue type and surrounding tissue. Physiology simulation contains the used phantom configuration, the corresponding tissuecompartment model, the applied flow profile and range, and the simulation of motion (e.g., breathing and cardiac motion). Pathology simulation indicates the presence of perfusion deficit simulation.

Extracted parameters for the studied PI application encounters the used contrast protocol, imaging system, and $\mathrm{BF}$ model. We also listed the studied input and output variables for the diverse phantom applications. Input variables were categorised as follows: (1) phantom/patient characteristics; (2) contrast protocol, if applicable; (3) imaging method; and (4) flow quantification method (see Fig. 1). Output variables included the following perfusion measures: arterial input function; tissue response function; MTT; BV; and BF. If mentioned by the authors, we listed published results on phantom performance, which describes the relation between the "ground truth" flow measure and the obtained quantitative PI outcomes. Finally, we documented in which studies phantom data are compared with human, animal, or mathematical data, and which phantoms are commercially available.

\section{Results}

\section{Phantom data assessment}

We have retrieved 463 articles using Scopus, of which 397 were rejected after abstract screening and another 32 after full-text reading. The search resulted in 37 accepted articles including cross-references (Fig. 2). Tables 1, 2, 3, and 4 summarise our main findings on phantom designs and applications in diverse PI domains. 


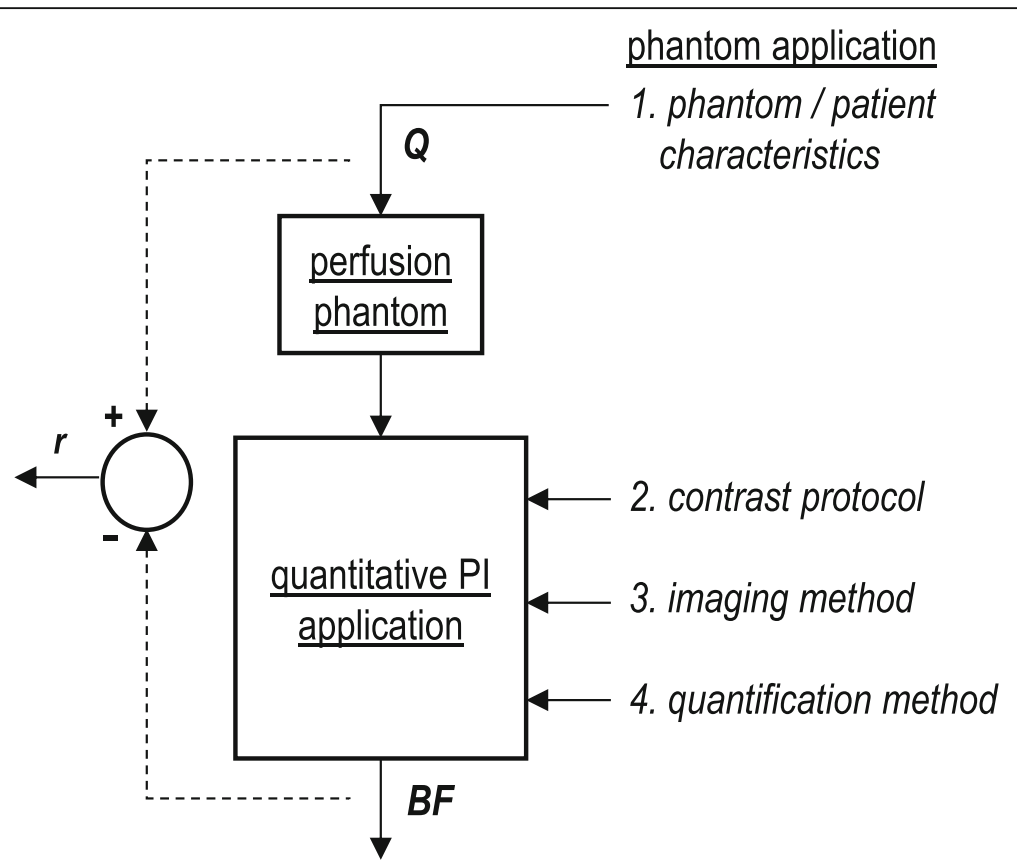

Fig. 1 System representation of ground truth validation process of quantitative perfusion imaging (PI). The diverse input variables that might affect quantitative perfusion outcomes are shown on the right. $Q$ serves as an example input variable and refers to set phantom flow in $\mathrm{mL} / \mathrm{min}$. $B F$ is accordingly the computed blood flow in $\mathrm{mL} / \mathrm{min}$ (system output), and $r$ is the residual between both. The latter can be translated into a measure of accuracy. The figure summarises the central topics of this review paper

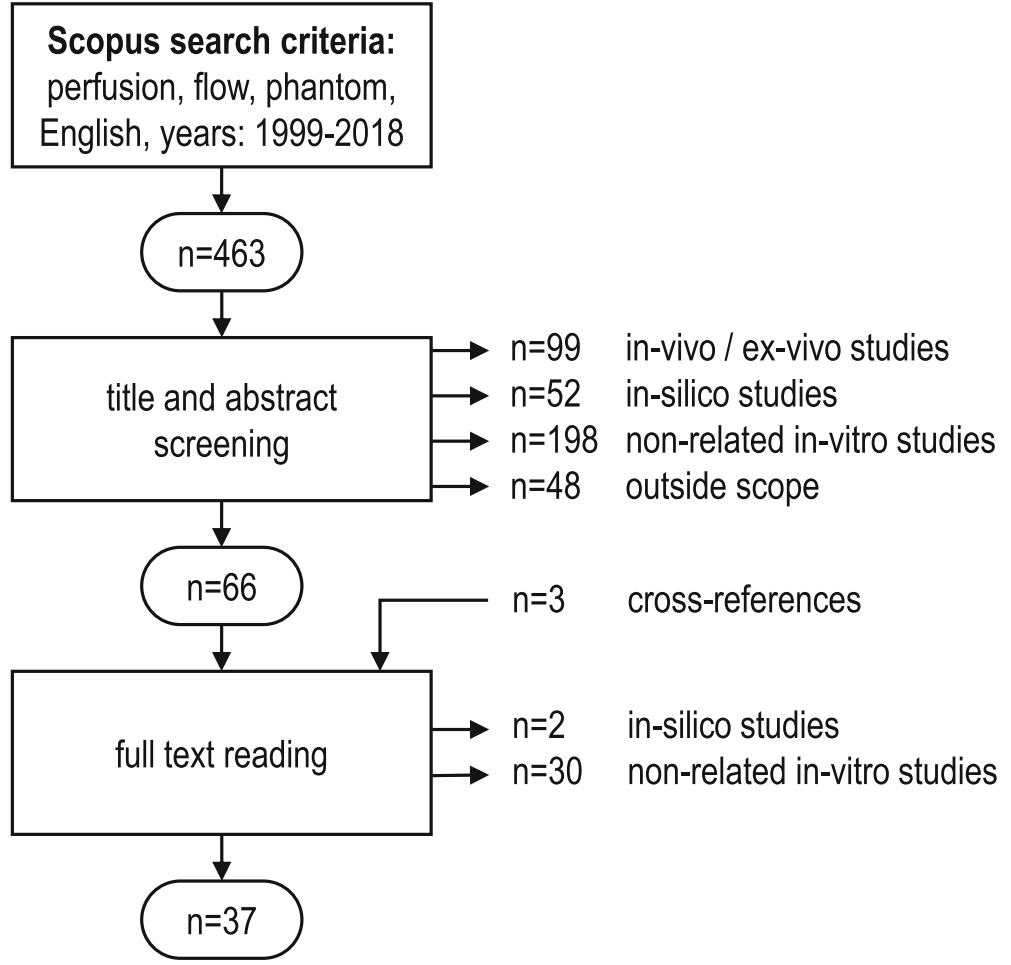

Fig. 2 Flow chart of study selection process 


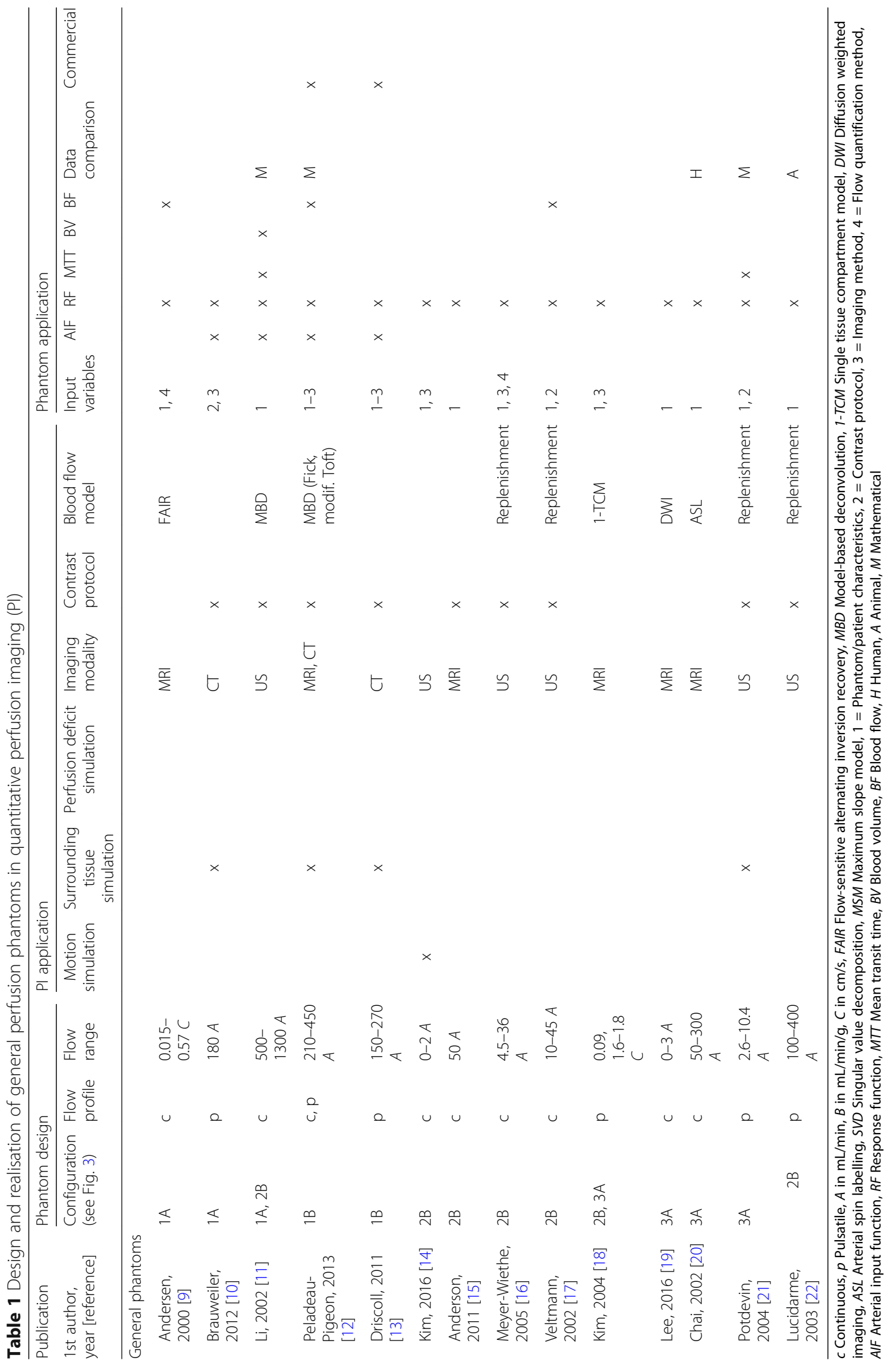









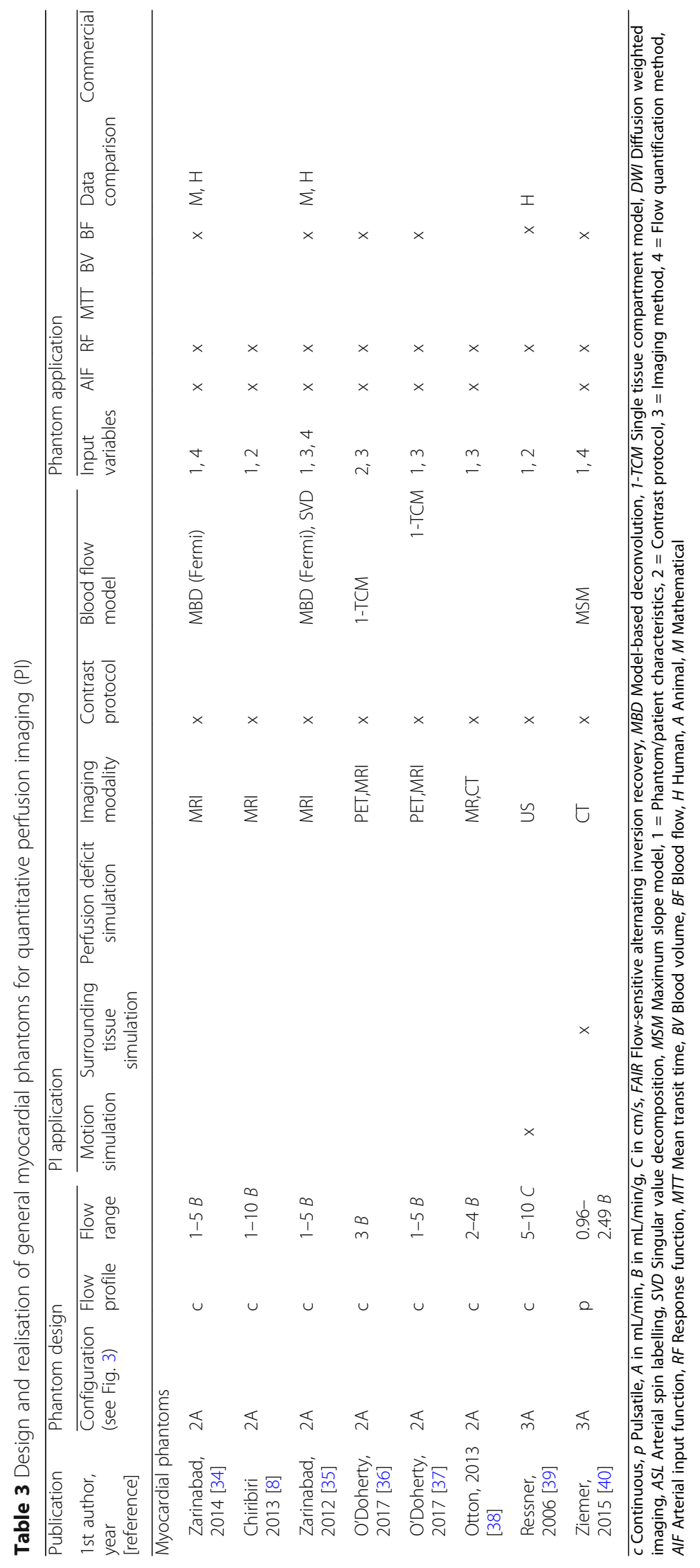




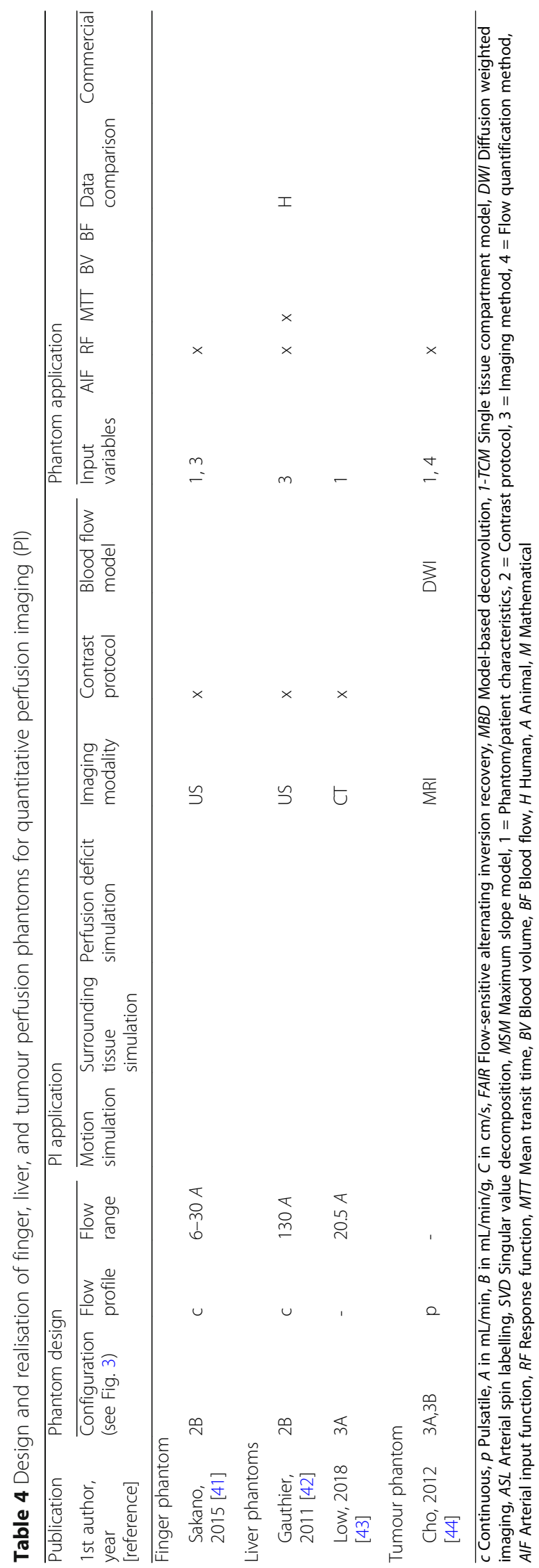




\section{Phantom design}

Anatomically, the phantoms simulate perfusion of various tissue types, including organ specific tissue (brain, $n=11$ articles; myocardial, $n=8$; liver, $n=2$; tumour, $n=1$; finger, $n=1)$ and non-specific tissue $(n=14)$. Several phantoms additionally mimic surrounding tissue (Tables 1,2 , 3 , and 4). All phantoms comprise a simplified "physiologic" model of perfusion that can be translated into a single tissue compartment model. Figure 3 schematically illustrates the basics of six distinguished phantom configurations, which specify three phantom types: basic $(n=6$ articles); aligned capillaries $(n=22)$; and tissue filled $(n=$ 12). The observed phantom designs simulate the microvasculature and tissue as one combined volume $(n=23$ articles) or two physically separated volumes $(n=17)$ (e.g., via a semipermeable membrane). Note that papers can present more than one phantom, and phantom designs may slightly differ from the schematic representations.

Basic phantoms generally consist of a single volume with ingoing and outgoing tubes, disregarding physiological simulation of microcirculation and tissue. In capillary phantoms, the microvasculature is simulated as a volume filled with unidirectional aligned hollow fibres or straws (e.g., a dialysis cartridge). The amount, diameter, and permeability of these fibres vary. Tissue-filled phantoms incorporate tissuemimicking material inside the volume, which subsequently leads to formation of a "microvasculature". Used materials include sponge [20, 21, 33, 44], (micro)beads $[19,31,40]$, gel $[18,39]$, and printed microchannels [32, 43]. Remarkably, in most studies, fluid exchange between simulated microvasculature and tissue (i.e., transfer rates $K_{1}$ and $k_{2}$ ) was uncontrollable, except for the study performed by Ohno et al. [33]. In this study, the compliance of the capacitor space could be altered to control $k_{2}$ to some extent. Low et al. [43] and Ebrahim et al. [32] have mathematically simulated the desired phantom flow configuration, before printing the microchannels. However, these models did not simulate fluid exchange between microvasculature and tissue. Continuous flow was applied in 26 phantom studies and pulsatile/peristaltic flow in 11 phantom studies. Flow settings vary per study and target organ and are presented in three different units (Tables 1, 2, 3, and 4). In case of brain and myocardial perfusion phantom modelling, flow experiments do not always cover the whole physiological range (Fig. 4). In addition, we observed two phantom studies that incorporated clutter motion (i.e., small periodic motion), but no studies included breathing or cardiac motion (Tables 1, 2, 3, and 4).

\section{1-tissue compartment model}



perfusion phantom configurations

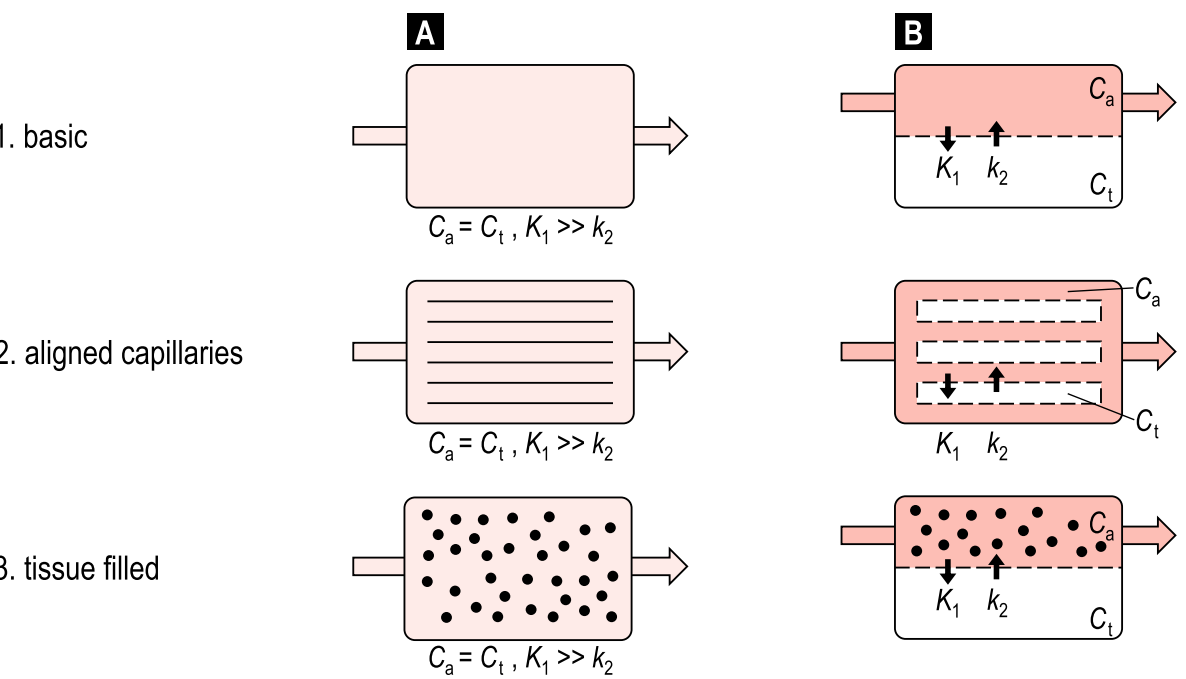

Fig. 3 Schematic representation of the 1-tissue compartment model and six derived phantom configurations. A distinction is made between three phantom types: basic, aligned capillaries and tissue filled (black spheres). Moreover, the microvasculature and tissue can be simulated as one combined (a) or two separated volumes (b) (e.g., via a porous membrane). $C_{p}$ and $C_{t}$ represent the concentration of the compound of interest (is being imaged) in the simulated blood plasma and tissue, respectively. $K_{1}$ and $k_{2}$ comprise the two transfer coefficients. Formation of in- and outgoing flow (arrow) and compartment flow varies per individual phantom design 


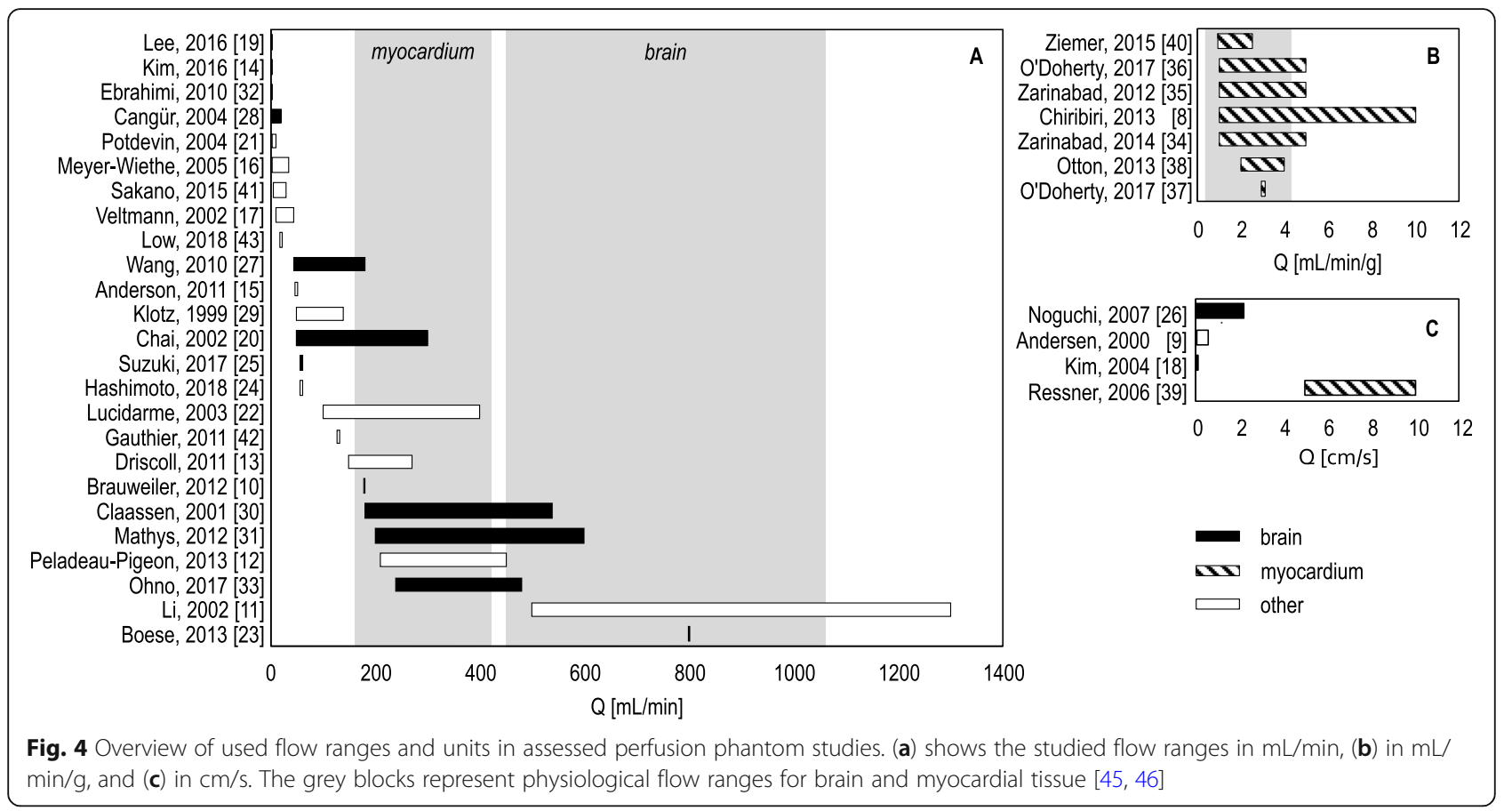

Regional perfusion deficit simulation (pathology) was only executed by Boese et al. [23]. Several studies mimicked some sort of global perfusion deficits by reducing the total flow or perfusion rate.

\section{Studied PI applications}

Tables 1, 2, 3, and 4 depict 17 studies focusing on MRI, 11 on ultrasound imaging, 11 on CT, and 2 on PET; 4 studies presented a direct comparison of MRI with PET or CT. A contrast-enhanced protocol was used in 28 studies. The used BF model for perfusion quantification varies per imaging modality and contrast protocol.

\section{Phantom applications}

Variables related to phantom/patient characteristics $(n=$ $32)$, contrast protocol $(n=12)$, imaging method $(n=$ $16)$, and quantification method $(n=7)$ were studied in relation to various quantitative perfusion measures (Table 1). Most papers describe the influence of flow settings on quantitative perfusion outcomes, followed by variation in contrast volume and acquisition protocol. Several studies compare outcomes to human/patient data $(n=7)$, animal data $(n=2)$, and mathematical simulations $(n=9)$ (Table 1$)$. In addition, we have identified one commercially available perfusion phantom that is described by Driscoll et al. [13] and applied by PeladeauPigeon et al. [12]. The relation between the "ground truth" flow measure and quantitative PI outcomes is summarised in Table 5. Remarkable is the diversity in used measures of perfusion and comparison (e.g., absolute errors, correlations statistics).

\section{Discussion}

A systematic search of the literature (from 1999 to 2018) was performed on contemporary perfusion phantoms. Detailed information was provided on three main aspects for ground truth evaluation of quantitative PI applications. We have elaborated on thirty-seven phantom designs, whereby focusing on anatomy, physiology and pathology simulation. In addition, we have listed the imaging system, contrast protocol and BF model for the studied PI applications. Finally, we have documented for each phantom application the investigated input and output variables, data comparison efforts and commercial availability. Hence, this review presents as main result an overview on perfusion phantom approaches and emphasises on the choices and simplifications in phantom design and realisation.

Although perfusion phantom modelling involves various tissues and applies to divers PI applications, we observe similarities in overall phantom designs and configurations. These configurations can be categorised in three types (6/40 basic, $22 / 40$ capillary, and $12 / 40$ tissue filled) and two representations of microvasculature and tissue $(23 / 40$ as one combined and $17 / 40$ as two separated compartments). Differences in these six phantom configurations are reflected in the resulting flow dynamics, e.g., how a contrast material is distributed and how long it stays inside the simulated organ tissue. None of the assessed phantoms could control inter-compartmental fluid exchange. Ideally, one would be able to fine-tune the exact flow dynamics in perfusion phantom modelling to achieve 
Table 5 Design and realisation of brain perfusion phantoms for quantitative perfusion imaging (PI)

\begin{tabular}{|c|c|c|c|}
\hline 1st author, year [reference] & Perfusion measure(s) & Phantom performance & Q \\
\hline \multicolumn{4}{|l|}{ Direct comparison with Q } \\
\hline Klotz, 1999 [29] & $\mathrm{BF}$ & $r=0.990$ & $50-140 \mathrm{~mL} / \mathrm{min}$ \\
\hline Wang, 2010 [27] & $\mathrm{BF}$ & $r>0.834$ & $45-180 \mathrm{~mL} / \mathrm{min}$ \\
\hline Mathys, 2012 [31] & $\mathrm{BF}$ & $r=0.995$ & $200-600 \mathrm{~mL} / \mathrm{min}$ \\
\hline Peladeau-Pigeon, 2013 [12] & $\mathrm{BF}$ & $r=0.992$ & $210-450 \mathrm{~mL} / \mathrm{min}$ \\
\hline Ohno, 2017 [33] & $\mathrm{BF}$ & $r>0.90$ & $240-480 \mathrm{~mL} / \mathrm{min}$ \\
\hline Ziemer, 2015 [40] & $\mathrm{BF}$ & $r=0.98$ & $0.96-2.49 \mathrm{~mL} / \mathrm{g} / \mathrm{min}$ \\
\hline O'Doherty, 2017 [36] & $\mathrm{BF}$ & $r=0.99$ & $1-5 \mathrm{~mL} / \mathrm{g} / \mathrm{min}$ \\
\hline Andersen, 2000 [9] & $\mathrm{BF}$ & $\begin{array}{l}\varepsilon \approx 0.015 \pm 0.03 \mathrm{~cm} / \mathrm{s} \\
\varepsilon \approx 0.001 \pm 0.03 \mathrm{~cm} / \mathrm{s}\end{array}$ & $\begin{array}{l}0.015 \pm 0.002 \mathrm{~cm} / \mathrm{s} \\
0.570 \pm 0.003 \mathrm{~cm} / \mathrm{s}\end{array}$ \\
\hline Ressner, 2006 [39] & $\mathrm{BF}$ & $\begin{array}{l}\varepsilon>40 \% \\
\varepsilon<20 \%\end{array}$ & $\begin{array}{l}1-3 \mathrm{~cm} / \mathrm{s} \\
5-7 \mathrm{~cm} / \mathrm{s}\end{array}$ \\
\hline Zarinabad, 2012 [35] & $\mathrm{BF}$ & $\begin{array}{l}\varepsilon=0.007 \pm 0.002 \mathrm{~mL} / \mathrm{g} / \mathrm{min} \\
\varepsilon=0.23 \pm 0.26 \mathrm{ml} / \mathrm{g} / \mathrm{min}\end{array}$ & $\begin{array}{l}0.5 \mathrm{~mL} / \mathrm{g} / \mathrm{min} \\
5 \mathrm{~mL} / \mathrm{g} / \mathrm{min}\end{array}$ \\
\hline Zarinabad, 2014 [34] & $\mathrm{BF}$ & $\begin{array}{l}\varepsilon<0.03 \mathrm{~mL} / \mathrm{g} / \mathrm{min} \\
\varepsilon<0.05 \mathrm{ml} / \mathrm{g} / \mathrm{min}\end{array}$ & $\begin{array}{l}2.5-5 \mathrm{~mL} / \mathrm{g} / \mathrm{min} \\
1-2.5 \mathrm{~mL} / \mathrm{g} / \mathrm{min}\end{array}$ \\
\hline Suzuki, 2017 [25] & $\mathrm{BF}$ & $\varepsilon \approx 0.0589 \pm 0.0108 \mathrm{~mL} / \mathrm{g} / \mathrm{min}$ & $0.1684 \mathrm{~mL} / \mathrm{g} / \mathrm{min}$ \\
\hline Hashimoto, 2018 [24] & $\mathrm{BF}$ & $\varepsilon \approx 0.0446 \pm 0.0130 \mathrm{~mL} / \mathrm{g} / \mathrm{min}$ & $0.1684 \mathrm{~mL} / \mathrm{g} / \mathrm{min}$ \\
\hline Ebrahimi, 2019 [32] & $\mathrm{BF}$ & $\mathrm{BF} / \mathrm{Q}>0.6$ & $0.12-1.2 \mathrm{~mL} / \mathrm{min}$ \\
\hline \multicolumn{4}{|l|}{ Indirect comparison with $\mathrm{Q}$} \\
\hline Veltmann, 2002 [17] & $r_{\text {kin }}$ & $r>0.984, x^{2}<0.019$ & $10-45 \mathrm{~mL} / \mathrm{min}$ \\
\hline Chai, 2002 [20] & $\Delta S I$ ratio & $r=0.995$ & $50-300 \mathrm{~mL} / \mathrm{min}$ \\
\hline Cangür, 2004 [28] & $\begin{array}{l}\text { TTP } \\
\text { PSI } \\
\text { AUC } \\
\text { PG } \\
\text { FWHM }\end{array}$ & $\begin{aligned} r & =-0.964 \\
r & =0.683 \\
r & =0.668 \\
r & =0.907 \\
r & =-0.63\end{aligned}$ & $1.8-21.6 \mathrm{~mL} / \mathrm{min}$ \\
\hline Myer-Wiethe, 2005 [16] & $\Delta S I$ & $r=0.99$ & $4.5-36 \mathrm{~mL} / \mathrm{min}$ \\
\hline Lee, 2016 [19] & $f_{\mathrm{p}}$ & $r>0.838$ & $1-3 \mathrm{~mL} / \mathrm{min}$ \\
\hline O'Doherty, 2017 [36] & $\mathrm{SI}$ & $\begin{array}{l}r=0.99 \\
r=0.99\end{array}$ & $\begin{array}{l}1-5 \mathrm{~mL} / \mathrm{g} / \mathrm{min} \text { (MRI) } \\
1.2-5.1 \mathrm{~mL} / \mathrm{g} / \mathrm{min} \text { (MRI vs PET) }\end{array}$ \\
\hline Kim, 2016 [14] & AUC & Efficiency $<50 \%$ & $0.1-2.0 \mathrm{~mL} / \mathrm{min}$ \\
\hline Claassen, 2001 [30] & AUC, PSI, MTT & No clear correlation with $\mathrm{Q}$ & \\
\hline
\end{tabular}

Phantom performance is predominantly listed in correlation statistics $\left(r, X^{2}\right)$ and absolute errors $(\varepsilon)$. A distinction is made between direct and indirect comparison with a "ground truth" flow measure $(\mathrm{Q})$, which consists of theoretical or experimental values. BF Blood flow, TTP Time to peak, MTT Mean transit time, AUC Area under the curve, $(P) S /$ Peak signal intensity, $f_{\mathrm{p}}$ Perfusion fraction, $r_{\text {kin }}$ Replenishment kinetics, FWHM Full width at half maximum, $P G$ Positive gradient

patient realistic (and contrast material specific) response function simulation. The required level of representativeness depends on the intended analyses, being closely related to the input parameters and boundary conditions of the BF model used. Since all assessed phantoms are limited to single tissue compartment models, phantom validation of higher order BF models should be performed with caution. It is generally important to verify whether assumptions in phantom modelling are justified for the intended phantom application. This also concerns decisions regarding motion, pulsatile flow and perfusion deficit simulation. For example, in myocardial perfusion modelling it could be relevant to incorporate respiratory and cardiac motion for certain analyses $[47,48]$, while for other tissues "motion" could be disregarded more easily.

The need for standardisation and validation of (quantitative) PI applications is widely recognised $[49,50]$. Perfusion phantom studies contribute to this endeavour, since these studies enable direct comparison between imaging systems and protocols. We only observed one commercial perfusion phantom in our search result. We foresee an increased clinical impact when phantoms become validated and widely available. In our opinion, phantom validation efforts are sometimes reported insufficiently and ambiguously. The concept of phantom validation can be difficult, since it is application- 
dependent and prone to subjectivity. The latter becomes apparent in the use of the words "considered", "reasonable", and "acceptable" (by whom, to whom, according to which criteria?) [51]. We therefore suggest to use Sargent's theory on model verification and validation [52]. Van Meurs' interpretation of this theory, including a practical checklist, is also applicable to physical, biomedical models (in adjusted form) [51]. For example, according to the checklist, investigators should verify whether the applied flow range covers the full physiological range. Our results (see Fig. 4) show great diversity in measured flow ranges. In addition, investigators are advised to consult physiologists and clinicians along the process, and compare findings with clinical data. In nine studies, phantom data are indeed compared with human or animal perfusion data (see Table 1).

When analysing phantom results, we noticed that investigators use different measures to evaluate quantitative PI outcomes, which hampers comparability (see Table 5). Some investigators express the relation between quantitative PI outcomes and the "ground truth" flow in correlation statistics or plots and others in absolute errors. Due to the diversity in outcome measures, applied flow ranges, and amount of measurements carried out, interpretation of these results should be handled with caution. A uniform, unambiguous measure to evaluate both phantom validity and the accuracy and precision of quantitative PI outcomes is desired.

This study has limitations. Our search was limited to articles published between 1999 and 2018, yet we are aware that the development and use of perfusion phantoms date further back. Contemporary studies build on these designs, which makes it relevant to elaborate on perfusion phantom experiments in advanced PI systems. Furthermore, we have decided to leave out detailed information on phantom design and fabrication (e.g., material choices and dimensions), since this information can be found in the appropriate references. Besides, phantom manufacturing is highly subject to change. We expect to see more three-dimensional printed perfusion phantoms in the coming years $[43,53,54]$.

In conclusion, this systematic review provided insights into contemporary perfusion phantom approaches, which can be used for ground truth evaluation of quantitative PI applications. It is desirable to indicate an unambiguous measure for phantom validity. Furthermore, investigators in the field are recommended to perform measurements in the full physiological flow range, consult physiologists and clinicians along the process, and compare findings with clinical data. In this way, one can verify and validate whether made choices and simplifications in perfusion phantom modelling are justified for the intended application, hence increasing clinical impact.

\section{Abbreviations}

BF: Blood flow; BV: Blood volume; CT: Computed tomography; MRI: Magnetic resonance imaging; MTT: Mean transit time; PET: Positron emission tomography; PI: Perfusion imaging; Q: "Ground truth" flow measure

\section{Acknowledgements}

We acknowledge fruitful discussions with Willem van Meurs, PhD, regarding phantom modelling and validation.

\section{Authors' contributions}

All authors have made substantial contributions to the design of this review paper. MEK has performed the systematic search, accompanied by MJWG in the paper selection process. MEK and CHS contributed to the interpretation of data. Finally, all authors have drafted the work and substantively revised it. All authors read and approved the final manuscript.

\section{Funding}

No funding was received for this study.

\section{Availability of data and materials}

All data generated or analysed during this study are included in this published article.

Ethics approval and consent to participate

Not applicable.

\section{Consent for publication}

Not applicable

\section{Competing interests}

All authors declare not to have any financial or other relationships that could be seen as a competing interests.

\section{Author details}

${ }^{1}$ Multimodality Medical Imaging M3i Group, Faculty of Science and Technology, Technical Medical Centre, University of Twente, PO Box 217, Enschede, The Netherlands. ${ }^{2}$ Robotics and Mechatronics Group, Faculty of Electrical Engineering, Mathematics, and Computer Science, Technical Medical Centre, University of Twente, Enschede, The Netherlands. ${ }^{3}$ Medical Imaging Center, Department of Nuclear Medicine and Molecular Imaging, University Medical Center Groningen, University of Groningen, Groningen, The Netherlands. ${ }^{4}$ Biomedical Photonic Imaging Group, Faculty of Science and Technology, Technical Medical Centre, University of Twente, Enschede, The Netherlands.

Received: 28 March 2019 Accepted: 8 November 2019

Published online: 04 March 2020

References

1. Barbier EL, Lamalle L, Decorps M (2001) Methodology of brain perfusion imaging. J Magn Reson Imaging. 13:496-520. https://doi.org/10.1002/jmri. 1073

2. Eck BL, Muzic RF, Levi J et al (2018) The role of acquisition and quantification methods in myocardial blood flow estimability for myocardial perfusion imaging CT. Phys Med Biol. 63:185011. https://doi.org/10.1088/ 1361-6560/aadab6

3. Bengel FM (2011) Leaving relativity behind. J Am Coll Cardiol. 8:749-751. https://doi.org/10.1016/j.jacc.2011.02.068

4. Sviri GE, Britz GW, Lewis DH, Newell DW, Zaaroor M, Cohen W (2006) Dynamic perfusion computed tomography in the diagnosis of cerebral vasospasm. Neurosurgery 59:319-324. https://doi.org/10.1227/01.NEU. 0000222819.18834 .33

5. Morton G, Chiribiri A, Ishida M et al (2012) Quantification of absolute myocardial perfusion in patients with coronary artery disease. J Am Coll Cardiol 60:1546-1555. https://doi.org/10.1016/j.jacc.2012.05.052

6. Slomka P, Xu Y, Berman D, Germano G (2014) Quantitative analysis of perfusion studies: strengths and pitfalls. J Nucl Cardiol 71:3831-3840. https://doi.org/10.1007/s12350-011-9509-2

7. Burrell S, MacDonald A (2006) Artifacts and pitfalls in myocardial perfusion imaging artifacts and pitfalls in myocardial perfusion imaging. J Nucl Med Technol 34:193-212 
8. Chiribiri A, Schuster A, Ishida M et al (2013) Perfusion phantom: an efficient and reproducible method to simulate myocardial first-pass perfusion measurements with cardiovascular magnetic resonance. Magn Reson Med 69:698-707. https://doi.org/10.1002/mrm.24299

9. Andersen IK, Sidaros K, Gesmar H, Rostrup E, Larsson HB (2000) A model system for perfusion quantification using FAIR. Magn Reson Imaging. 18: 565-574. https://doi.org/10.1016/S0730-725X(00)00136-3

10. Brauweiler R, Eisa F, Hupfer M, Nowak T, Kolditz D, Kalender WA (2012) Development and evaluation of a phantom for dynamic contrast-enhanced imaging. Invest Radiol. 47:462-467. https://doi.org/10.1097/RLI. 0b013e318250a72c

11. Li P-C, Yeh C-K, Wang S-W (2002) Time-intensity-based volumetric flow measurements: an in vitro study. Ultrasound Med Biol. 28:349-358. https:/ doi.org/10.1016/S0301-5629(01)00516-6

12. Peladeau-Pigeon M, Coolens C (2013) Computational fluid dynamics modelling of perfusion measurements in dynamic contrast-enhanced computed tomography: Development, validation and clinical applications. Phys Med Biol. 58:6111-6131. https://doi.org/10.1088/0031-9155/58/17/6111

13. Driscoll B, Keller H, Coolens C (2011) Development of a dynamic flow imaging phantom for dynamic contrast-enhanced CT. Med Phys. 38:48664880. https://doi.org/10.1118/1.3615058

14. Kim M, Abbey CK, Insana MF (2016) Efficiency of U.S. tissue perfusion estimators. IEEE Trans Ultrason Ferroelectr Freq Control. 63:1131-1139. https://doi.org/10.1109/TUFFC.2016.2571979

15. Anderson JR, Ackerman JJH, Garbow JR (2011) Semipermeable hollow fiber phantoms for development and validation of perfusion-sensitive MR methods and signal models. Concepts Magn Reson Part B Magn Reson Eng. 39B:149-158. https://doi.org/10.1002/cmr.b.20202

16. Meyer-Wiethe $\mathrm{K}$, Cangür $\mathrm{H}$, Seidel $\mathrm{G}$ (2005) Comparison of different mathematical models to analyze diminution kinetics of ultrasound contrast enhancement in a flow phantom. Ultrasound Med Biol. 31:93-98. https:// doi.org/10.1016/j.ultrasmedbio.2004.05.006

17. Veltmann C, Lohmaier S, Schlosser T et al (2002) On the design of a capillary flow phantom for the evaluation of ultrasound contrast agents at very low flow velocities. Ultrasound Med Biol. 28:625-634. https://doi.org/10. 1016/50301-5629(02)00499-4

18. Kim EJ, Kim DH, Lee SH, Huh YM, Song HT, Suh JS (2004) Simultaneous acquisition of perfusion and permeability from corrected relaxation rates with dynamic susceptibility contrast dual gradient echo. Magn Reson Imaging. 22:307-314. https://doi.org/10.1016/j.mri.2004.01.012

19. Lee JH, Cheong H, Song J-A et al (2016) Perfusion assessment using intravoxel incoherent motion-based analysis of diffusion-weighted magnetic resonance imaging. Invest Radiol. 51:520-528. https://doi.org/10.1097/RLI. 0000000000000262

20. Chai JW, Chen JH, Kao YH et al (2002) Spoiled gradient-echo as an arterial spin tagging technique for quick evaluation of local perfusion. J Magn Reson Imaging. 16:51-59. https://doi.org/10.1002/jmri.10128

21. Potdevin TC, Fowlkes JB, Moskalik AP, Carson PL (2004) Analysis of refill curve shape in ultrasound contrast agent studies. Med Phys. 31:623-632 https://doi.org/10.1118/1.1649534

22. Lucidarme O, Franchi-abella S, Correas J, Bridal SL, Kurtisovski E (2003) Blood flow quantification with contrast-enhanced US : "Entrance in the Section" phenomenon — phantom and rabbit study. Radiology. 228:473-479. https://doi.org/10.1148/radiol.2282020699

23. Boese A, Gugel S, Serowy S et al (2012) Performance evaluation of a C-Arm CT perfusion phantom. Int J Comput Assist Radiol Surg. 8:799-807. https:// doi.org/10.1007/s11548-012-0804-4

24. Hashimoto H, Suzuki K, Okaniwa E, limura H, Abe K, Sakai S (2017) The effect of scan interval and bolus length on the quantitative accuracy of cerebral computed tomography perfusion analysis using a hollow-fiber phantom. Radiol Phys Technol. 11:13-19. https://doi.org/10.1007/s12194-017-0427-0

25. Suzuki K, Hashimoto H, Okaniwa E et al (2017) Quantitative accuracy of computed tomography perfusion under low - dose conditions, measured using a hollow - fiber phantom. Jpn J Radiol. 35:373-380. https://doi.org/10. 1007/s11604-017-0642-y

26. Noguchi T, Yshiura T, Hiwatashi A et al (2007) Quantitative perfusion imaging with pulsed arterial spin labeling: a phantom study. Magn Reson Med Sci. 6:91-97. https://doi.org/10.2463/mrms.6.91

27. Wang Y, Kim SE, Dibella EVR, Parker DL (2010) Flow measurement in MRI using arterial spin labeling with cumulative readout pulses - theory and validation. Med Phys. 37:5801-5810. https://doi.org/10.1118/1.3501881
28. Cangür H, Meyer-Wiethe K, Seidel G (2004) Comparison of flow parameters to analyse bolus kinetics of ultrasound contrast enhancement in a capillary flow model. Ultraschall Med. 25:418-421. https://doi.org/10.1055/s-2004813796

29. Klotz E, König M (1999) Perfusion measurements of the brain: using dynamic CT for the quantitative assessment of cerebral ischemia in acute stroke. Eur J Radiol. 30:170-184. https://doi.org/10.1016/S0720048X(99)00009-1

30. Claassen L, Seidel G, Algermissen C (2001) Quantification of flow rates using harmonic grey-scale imaging and an ultrasound contrast agent: an in vitro and in vivo study. Ultrasound Med Biol. 27:83-88. https://doi.org/10.1016/ S0301-5629(00)00324-0

31. Mathys C, Rybacki K, Wittsack HJ et al (2012) A phantom approach to interscanner comparability of computed tomographic brain perfusion parameters. J Comput Assist Tomogr. 36:732-738. https://doi.org/10.1097/ rct.0b013e31826801df

32. Ebrahimi B, Swanson SD, Chupp TE (2010) A microfabricated phantom for quantitative MR perfusion measurements: validation of singular value decomposition deconvolution method. IEEE Trans Biomed Eng. 57:27302736. https://doi.org/10.1109/TBME.2010.2055866

33. Ohno N, Miyati T, Chigusa T et al (2015) Technical note: development of a cranial phantom for assessing perfusion, diffusion, and biomechanics. Med Phys 44:1646-54 . https://doi.org/10.1002/mp.12182

34. Zarinabad N, Hautvast GLTF, Sammut E et al (2014) Effects of tracer arrival time on the accuracy of high-resolution (Voxel-wise) myocardial perfusion maps from contrast-enhanced first-pass perfusion magnetic resonance. IEEE Trans Biomed Eng. 61:2499-2506. https://doi.org/10.1109/TBME.2014. 2322937

35. Zarinabad N, Chiribiri A, Hautvast GLTF et al (2012) Voxel-wise quantification of myocardial perfusion by cardiac magnetic resonance. Feasibility and Methods Comparison. Magn Reson Med. 68:1994-2004. https://doi.org/10. 1002/mrm.24195

36. O'Doherty J, Chalampalakis Z, Schleyer P, Nazir MS, Chiribiri A, Marsden PK (2017) The effect of high count rates on cardiac perfusion quantification in a simultaneous PET-MR system using a cardiac perfusion phantom. EJNMMI Phys. 4:31. https://doi.org/10.1186/s40658-017-0199-y

37. O'Doherty J, Sammut E, Schleyer P et al (2017) Feasibility of simultaneous PET-MR perfusion using a novel cardiac perfusion phantom. Eur J Hybrid Imaging. 1:1-14. https://doi.org/10.1186/s41824-017-0008-9

38. Otton J, Morton G, Schuster A et al (2013) A direct comparison of the sensitivity of $\mathrm{CT}$ and $\mathrm{MR}$ cardiac perfusion using a myocardial perfusion phantom. J Cardiovasc Comput Tomogr. 7:117-124. https://doi.org/10.1016/ j.jcct.2013.01.016

39. Ressner M, Brodin LA, Jansson T, Hoff L, Ask P, Janerot-Sjoberg B (2006) Effects of ultrasound contrast agents on doppler tissue velocity estimation. J Am Soc Echocardiogr. 19:154-164. https://doi.org/10.1016/j.echo.2005.09.025

40. Ziemer BP, Hubbard L, Lipinski J, Molloi S (2015) Dynamic CT perfusion measurement in a cardiac phantom. Int J Cardiovasc Imaging. 31:14511459. https://doi.org/10.1007/s10554-015-0700-4

41. Sakano R, Kamishima T, Nishida M, Horie T (2015) Power Doppler signal calibration between ultrasound machines by use of a capillary-flow phantom for pannus vascularity in rheumatoid finger joints: a basic study. Radiol Phys Technol.8:120-124. https://doi.org/10.1007/s12194-014-0299-5

42. Gauthier TP, Averkiou MA, Leen ELS (2011) Perfusion quantification using dynamic contrast-enhanced ultrasound: the impact of dynamic range and gain on time-intensity curves. Ultrasonics. 51:102-106. https://doi.org/10. 1016/j.ultras.2010.06.004

43. Low L, Ramadan S, Coolens C, Naguib HE (2018) 3D printing complex lattice structures for permeable liver phantom fabrication. Bioprinting. 10. https:// doi.org/10.1016/j.bprint.2018.e00025

44. Cho GY, Kim S, Jensen JH, Storey P, Sodickson DK, Sigmund EE (2012) A versatile flow phantom for intravoxel incoherent motion MRI. Magn Reson Med 67:1710-1720. https://doi.org/10.1002/mrm.23193

45. Murthy VL, Bateman TM, Beanlands RS et al (2017) Clinical quantification of myocardial blood flow using PET: joint position paper of the SNMMI Cardiovascular Council and the ASNC. J Nucl Med. 59:273-293. https://doi. org/10.2967/jnumed.117.201368

46. Brown RP, Delp MD, Lindstedt SL, Rhomberg LR, Beliles RP (1997) Physiological parameter values for physiologically based pharmacokinetic models. Toxicol Ind Health. 13:407-484. https://doi.org/10.1177/ 074823379701300401 
47. Chrysanthou-Baustert I, Polycarpou I, Demetriadou O et al (2017) Characterization of attenuation and respiratory motion artifacts and their influence on SPECT MP image evaluation using a dynamic phantom assembly with variable cardiac defects. J Nucl Cardiol. 24:698-707. https:// doi.org/10.1007/s12350-015-0378-y

48. Fieseler M, Kugel H, Gigengack F et al (2013) A dynamic thorax phantom for the assessment of cardiac and respiratory motion correction in PET/MRI: A preliminary evaluation. Nucl Instrum Methods Phys Res. 702:59-63. https:// doi.org/10.1016/..nima.2012.09.039

49. Leiva-Salinas C, Hom J, Warach S, Wintermark M (2012) Stroke Imaging Research Roadmap. Neuroimaging Clin N Am. 21:1-9. https://doi.org/10, 1016/j.nic.2011.01.009

50. Miles KA, Lee T, Goh V, Klotz E, Cuenod C (2012) Current status and guidelines for the assessment of tumour vascular support with dynamic contrast-enhanced computed tomography. Eur Radiol 22:1430-1441. https://doi.org/10.1007/s00330-012-2379-4

51. Van Meurs WL (2011) Modeling and simulation in biomedical engineering applications in cardiorespiratory physiology. The McGraw-Hill Companies. https://doi.org/10.1109/MPUL.2013.2289532

52. Sargent RG (2010) Verification and validation of simulation models. Proc 2010 Winter Simul Conf 166-183. https://doi.org/10.1109/WSC.2007.4419595

53. Wang K, Ho C, Zhang C, Wang B (2017) A review on the 3D printing of functional structures for medical phantoms and regenerated tissue and organ applications. Engineering. 3:653-662. https://doi.org/10.1016/J.ENG. 2017.05.013

54. Wood RP, Khobragade P, Ying L et al (2015) Initial testing of a 3D printed perfusion phantom using digital subtraction angiography. Proc SPIE 9417, Medical Imaging 2015: Biomedical Applications in Molecular, Structural, and Functional Imaging, 94170V. https://doi.org/10.1117/12.2081471

\section{Publisher's Note}

Springer Nature remains neutral with regard to jurisdictional claims in published maps and institutional affiliations.

\section{Submit your manuscript to a SpringerOpen ${ }^{\circ}$ journal and benefit from:}

- Convenient online submission

- Rigorous peer review

- Open access: articles freely available online

- High visibility within the field

- Retaining the copyright to your article

Submit your next manuscript at $\boldsymbol{\nabla}$ springeropen.com 